\title{
Functional Gastrointestinal Disorders Induced by Esophageal Atresia Surgery: Is It Valid in Humans?
}

\author{
Ugur Halac, ${ }^{1 *}$ Marine Revillion, ${ }^{2}$ Laurent Michaud, ${ }^{2}$ Frédéric Gottrand ${ }^{2}$ and Christophe Faure ${ }^{1}$ \\ ${ }^{1}$ Division of Gastroenterology, Hepatology and Nutrition, Department of Pediatrics, Sainte-Justine Hospital, Université de Montréal, Montréal, \\ Québec, Canada; and ${ }^{2}$ Division of Gastroenterology, Hepatology and Nutrition, and Reference Centre for Congenital and Malformative \\ Oesophageal Diseases, Department of Paediatrics, Jeanne de Flandre Children's Hospital and Faculty of Medicine, University Lille 2, Lille, France
}

\begin{abstract}
Background/Aims
Functional gastrointestinal disorders (FGID) affect 15\%-20\% of the general pediatric and adult population. Animal models suggest that a neonatal stress such as invasive procedures and maternal separation could be responsible for visceral hypersensitivity and FGID. We tested the hypothesis that congenital esophageal atresia (EA), a condition corrected during the neonatal period and associated with multiple stresses, is a clinically significant risk factor for the development of FGID later in life. We postulated that, to be clinically significant, the effect of neonatal stress on the incidence of FGID should be as strong as that of enteric infections in the development of irritable bowel syndrome in children.
\end{abstract}

\section{Methods}

Subjects with EA and healthy controls were enrolled in this multicenter cohort study. Gastrointestinal symptoms were assessed by a questionnaire and FGID was diagnosed using the Rome III criteria.

\section{Results}

Fifty-three children ( 25 girls; median age, 12 years) with EA were compared to 72 age- and sex-matched controls. Although 11 children with EA $(21 \%)$ had a FGID diagnosis versus 8 controls $(11 \%)$, this difference was not significant $\left(\chi^{2}=2.20, P\right.$ $>0.05)$. In subjects with EA, the presence of associated malformations, the occurrence of complications during the first month, and the length of hospital stay > 30 days did not influence the incidence of FGID. Chronic abdominal pain was present in $38 \%$ of subjects with EA versus $25 \%$ of controls $(P>0.05)$.

\section{Conclusions}

Neonatal stress secondary to surgical correction of EA is not a clinically significant risk factor for the development of FGID in childhood.

(J Neurogastroenterol Motil 2012;18:406-411)

Key Words

Esophageal atresia; Irritable bowel syndrome; Life change events

Received: June 20, 2012 Revised: August 18, 2012 Accepted: September 11, 2012

(c) This is an Open Access article distributed under the terms of the Creative Commons Attribution Non-Commercial License (http://creativecommons. org/licenses/by-nc/3.0) which permits unrestricted non-commercial use, distribution, and reproduction in any medium, provided the original work is properly cited.

*Correspondence: Ugur Halac, MD

Division of Pediatric Gastroenterology, Hepatology and Nutrition, Sainte-Justine Hospital, Université de Montréal, 3175

Côte-Sainte-Catherine, Montréal, Québec H3T 1C5, Canada

Tel: +1-5143454931(2562),Fax: +1-5143454999, E-mail: ugur.halac@umontreal.ca

Financial support: None.

Conflicts of interest: None. 


\section{Introduction}

Functional gastrointestinal disorders (FGID) are defined as recurrent symptoms that are not yet explainable by structural or biochemical anomalies. In children, the most frequent FGIDs include irritable bowel syndrome (IBS), functional abdominal pain (FAP) and functional dyspepsia (FD), which are defined by the symptom-based Rome criteria. ${ }^{1}$

The mechanisms underlying the pathophysiology of FGID are not unique and rather the result of various mechanisms. FGID may result from the interaction of multiple genetic, biologic, psychological and social factors. Among them, early-life adverse events in the neonatal period either somatic or psychological have been implicated as possible contributing factors. Animal studies have shown that the neonatal period is characterized by a very high susceptibility to stress including colonic irritation $^{2}$ and maternal deprivation ${ }^{3}$ which lead to visceral hypersensitivity in adulthood. ${ }^{2,3}$ The latter has been closely related not only to central ${ }^{2}$ but also to peripheral sensitization. ${ }^{3,4}$ In humans, studies have attempted to confirm these findings, suggesting the putative key role of birth weight $<1.5 \mathrm{~kg}$, repetitive gastric suctions in neonates, and surgical correction of pyloric stenosis. ${ }^{5-7}$

Esophageal atresia (EA) occurs in about 1 out of 3,000 live births. ${ }^{8}$ Surgical repair is obligatory and performed in the first day of life followed by a stay in the neonatal intensive care unit (NICU). Therefore, patients operated at birth for EA experience highly stressful neonatal events including surgery, painful procedures, gastric tube placement and NICU stays with prolonged maternal separation.

We hypothesized that subjects operated for EA (exposed group) were more prone to develope FGID and abdominal pain compared to control subjects without a complex neonatal history. In this multicenter cohort study, we sought to measure the incidence of abdominal pain and abdominal pain-related FGID (IBS, FD and FAP), using the Rome III criteria, in patients with congenital EA corrected during the neonatal period, and compared it to subjects without a complex neonatal history. We also aimed to determine if complications and events following surgery in the first month of life influence long-term development of abdominal pain-related FGID in childhood.

\section{Materials and Methods}

\section{Subjects}

All subjects with EA, aged 8 to 18 years, followed at the EA Clinics at Hôpital Sainte-Justine (Université de Montréal, Québec, Canada) and at Hôpital Jeanne de Flandre (University of Lille, Lille, France) were approached for inclusion. Exclusion criteria included a history of inflammatory bowel disease, an acute enteric infection (gastroenteritis) or an abdominal surgery in the previous 4 weeks, and an intellectual disability. Data was gathered from patients' charts using standardized data collection forms. We included patient demographics (sex, gestational age, birth weight, type of atresia and associated malformations), occurrence of post-operative complications in the first month of life (pneumothorax, chylothorax, anastomotic leak and early recurrent fistula), duration of intubation, age of oral feeding, duration of NICU stay and duration of hospital stay.

Controls $\geq 8$ years of age were enrolled using the same exclusion criteria from orthopedic clinics in each hospital. Control subjects had a benign neonatal course (absence of neonatal surgery and neonatal maternal separation) and no history of surgery for scoliosis.

\section{Methods}

All participants had to complete the Questionnaire of Pediatric Gastrointestinal Symptoms (QPGS). The QPGS is a questionnaire, validated in English and French, that assesses symptoms associated with FGID in children, and has been adapted to the pediatric Rome III criteria. ${ }^{1,9}$ This structured questionnaire includes sections assessing bowel habits, abdominal pain (pain duration and frequency), dysphagia, heartburn, regurgitations and data on limitations of activities (missed days of school and missed activities with friends due to pain). The questionnaire was completed by each child or with the help of a parent for children less than 10 years old. The primary outcome was the prevalence of abdominal pain-associated FGID (IBS, FD and FAP) according to Rome III criteria. The secondary outcome was the prevalence of chronic abdominal pain defined as abdominal pain reported either continuously or intermittently at least once a week for more than 8 weeks of duration.

The protocol was approved by the institutional ethics committees of the 2 hospitals, and appropriate consent was obtained from all participants. Consent was signed by the parents or legal 
guardian and by the child himself if older than 14 years of age.

\section{Sample Size Calculation and Statistical Anal- ysis}

We postulated that, to be clinically significant, the effect of neonatal stress on the incidence of abdominal pain-related FGID in the exposed subjects should be as strong as the effect of enteric infections in the development of IBS in childhood. ${ }^{10}$ Studies have shown that the prevalence of abdominal pain-related FGID in the general pediatric population is $10 \%-12 \%$ and increases to $30 \%-32 \%$ in those that have had a recent enteric infection (relative risk 3.2). ${ }^{10}$ We therefore hypothesized that 30\%-32\% of exposed subjects (with EA) should be affected by FGID and calculated that 52 patients and 52 controls were necessary in each cohort to achieve a uni-directional alpha of 0.05 with power of $80 \%$. The percentage of children who reported abdominal pain-related FGID in each group was compared and odds ratio was obtained. In the group of patients operated for EA (exposed group), further analysis was conducted between subjects with and without FGID: continuous variables (gestational age, birth weight, age at extubation, age at oral feeding, length of NICU stay and length of hospital stay) and dichotomous variables (presence of associated malformations, post-operative complications during the first month, and hospital stay duration $>30$ days) were compared.

Appropriate descriptive statistics were used to summarize the general features of the population and questionnaire findings. We performed comparisons between the groups using the Student's $t$ test and Wilcoxon test to compare means and medians for the continuous variables, and the $\chi^{2}$ test or Fisher exact test for discrete variables. Statistical significance was expressed as $P<0.05$ and SPSS software (version 18.0; SPSS Inc., Chicago, IL, USA) was used for all analyses.

\section{Results}

Fifty-five subjects with EA fulfilled the inclusion criteria. Fifty-three of them, 23 from Hôpital Ste-Justine and 30 from Jeanne de Flandres Hospital, (25 girls; median age, 12.3 years; range, 7.8-18.0) accepted to participate to the study. Seventy-two controls (34 girls; median age, 13.4 years; range, 7.8-18.5) were also included $(P>0.05$ for age and sex ratio of patients vs controls).

Based on the answers from the QPGS, 11 out of 53 children (21\%) with EA complained of FGID (IBS, $\mathrm{n}=5$; FAP, $\mathrm{n}=5$;
$\mathrm{FD}, \mathrm{n}=1)$ according to pediatric Rome III criteria compared to 8 out of 72 controls ( $11 \%$ ) (IBS, $\mathrm{n}=3$; FAP, $\mathrm{n}=3$; FD, $\mathrm{n}=2$ ) $\left(\chi^{2}=2.20, P>0.05\right)$. Odds Ratio of developing a FGID following EA repair was 2.09 (95\% CI, 0.77-5.64; $P>0.05$ ). There were no significant differences in symptom duration, intensity or limitation of activities, between exposed subjects and controls with FGID (Table 1).

In addition, we sought to determine if patient variables such as associated malformations, occurrence of post-operative complications, duration of intubation, age at oral feeding, duration of NICU stay or duration of hospital stay, which could all be con-

Table 1. Gastrointestinal Symptoms Severity Variables in Pediatric Subjects and Controls With Functional Gastrointestinal Disorders

\begin{tabular}{lll}
\hline & $\begin{array}{c}\text { Subjects with } \\
\text { FGID }(\mathrm{n}=11)\end{array}$ & $\begin{array}{c}\text { Controls with } \\
\text { FGID }(\mathrm{n}=8)\end{array}$ \\
\hline FGID type (n [\%]) & & \\
IBS & $5(45.0)$ & $3(37.5)$ \\
FAP & $5(45.0)$ & $2(25.0)$ \\
FD & $1(10.0)$ & $3(37.5)$ \\
Pain frequency (n [\%]) & & \\
$<$ Once/wk & $0(0.0)$ & $0(0.0)$ \\
Once/wk & $6(54.0)$ & $4(50.0)$ \\
Many times/wk & $4(36.0)$ & $3(37.5)$ \\
Everyday & $1(10.0)$ & $1(12.5)$ \\
Duration of pain (n [\%]) & & \\
2-3 mo & $2(18.0)$ & $2(25.0)$ \\
4-11 mo & $0(0.0)$ & $0(0.0)$ \\
$\geq 1$ yr & $9(82.0)$ & $6(75.0)$ \\
Duration of pain (n [\%]) & & $6(75.0)$ \\
$<1$ hr & $8(72.0)$ & $1(12.5)$ \\
1-2 hr & $2(18.0)$ & $0(0.0)$ \\
3-4 hr & $0(0.0)$ & $1(12.5)$ \\
Majority of the day & $0(0.0)$ & $0(0.0)$ \\
$\geq 1$ day & $1(10.0)$ & $0(100.0)$ \\
Quantification of pain (n [\%]) & & $0(0.0)$ \\
A little & $4(36.0)$ & $5(62.5)$ \\
From a little to a lot & $4(36.0)$ & $3(37.5)$ \\
A lot & $3(28.0)$ & $0(0.0)$ \\
Hugely & $0(0.0)$ & $0(0.0)$ \\
Missed days of school (n [\%]) & & \\
Never or < once/mo & $9(82.0)$ & \\
1-4 times/mo & $2(18.0)$ & \\
Everyday & $0(0.0)$ & \\
Missed social activities (n [\%]) & & \\
Never or $<$ once/mo & $9(82.0)$ & $(12.5)$ \\
1-4 times/mo & $2(18.0)$ & $(0.0)$ \\
Everyday & & \\
\hline
\end{tabular}

FGID, functional gastrointestinal disorders; IBS, irritable bowel syndrome; FAP, functional abdominal pain; FD, functional dyspepsia. 
Table 2. Distribution of Stress-related Factors in Pediatric Subjects Operated for Esophageal Atresia With or Without Functional Gastrointestinal Disorders

\begin{tabular}{lccc}
\hline & $\begin{array}{c}\text { EA with FGID } \\
(\mathrm{n}=11)\end{array}$ & $\begin{array}{c}\text { EA without FGID } \\
(\mathrm{n}=42)\end{array}$ & OR and $P$-value \\
\hline Gestational age (median [range], wk) & $38(33-42)$ & $38(29-40)$ & $P=0.912$ \\
Birth weight (median [range], g) & $2,400(1,465-3,270)$ & $2,835(1,050-3,750)$ & $P=0.123$ \\
Birth weight $<2,500 \mathrm{~g}$ (n [\%]) & $6(55)$ & $12(29)$ & OR $=0.36(0.77-11.72)$ \\
& & & $P=0.135$ \\
& $3(1-18)$ & $2(0-60)$ & $P=0.971$ \\
Age at extubation (median [range], days) & $6.5(0-19)$ & $7(2-26)$ & $P=0.872$ \\
Age at oral feeding (median [range], days) & $7(1-73)$ & $8(1-145)$ & $P=0.828$ \\
NICU stay duration (median [range], days) & $14.5(4-140)$ & $15.5(7-154)$ & OR $=2.67(0.62-11.48)$ \\
Hospital stay duration (median [range], days) & $8(72)$ & $21(50)$ & $P=0.177$ \\
Presence of associated malformations (n [\%]) & & & OR $=0.52(0.12-1.73)$ \\
& $4(36)$ & $22(52)$ & $P=0.344$
\end{tabular}

EA, esophageal atresia; FGID, functional gastrointestinal disorders; NICU, neonatal intensive care unit.

sidered as a proxy for assessing the intensity of neonatal stress, influencing the development of FGID. None of these parameters was a significant risk factor for the development of FGID in childhood and adolescence (Table 2).

Chronic abdominal pain as previously defined was present in 20/53 patients (38\%) and 18/72 controls (25\%) ( $P>0.05)$.

Compared to controls, the children with EA reported more frequent dysphagia (49\% vs $11 \%, P<0.001)$, heartburn $(25 \%$ vs $10 \%, P<0.05)$, regurgitations (23\% vs $8 \%, P<0.05)$, symptoms related to esophageal dysmotility and gastroesophageal reflux which were both associated to EA in the literature. ${ }^{12}$

\section{Discussion}

We showed that the neonatal stress induced by EA repair which involved many stressful events (repeated invasive procedures, maternal separation), was not a clinically significant risk factor for subsequent development of pain-associated FGID in later childhood. We have reported similar results even when less rigorous criteria of "chronic abdominal pain" were used. Furthermore, we showed that subjects suffering from FGID had not been exposed to more severe neonatal stressful conditions.

These results contrast with those of previously published human studies on consequences of neonatal stress on the occurrence of FGID. Indeed, a case-control retrospective study found that $22.4 \%$ of 108 patients hospitalized with FGID at a median age of 14 (range, 2-31) had experienced gastric suction at birth versus $11.2 \%$ in a control group. However, this retrospective study was prone to recall bias and gastric suction might thus be a confounding factor. ${ }^{5}$ A recent work by Saps \& Bonilla reported that infants operated for pyloric stenosis at a mean age of 39 days, developed at the age of 7.5 days, showed a significantly higher incidence of chronic abdominal pain (but not of FGID) compared to controls (OR, 4.27; 95\% CI, 1.43-12.70). ${ }^{7}$ Although the incidence of chronic abdominal pain was comparable to our present data $(25 \%$ in Saps \& Bonilla's study vs $38 \%$ in the present study), the incidence of abdominal pain was surprisingly very low (5\%) in the control group compared to the previously published data in children ${ }^{11,13}$ including those from this group. ${ }^{10}$

The strengths of the present work are that: (1) this is a prospectively designed multicenter study. We included a homogenous cohort of subjects with a history of severe neonatal stress who therefore represent an appropriate population to test the hypothesis of the role of early-life stress in the development of FGID; (2) the incidence of pain-related abdominal symptoms in control subjects is in agreement with previously published pediatric data suggesting that the control group was adequately selected ${ }^{10,11,13}$; and (3) the expected high incidence of dysphagia and gastroesophageal reflux symptoms in patients with operated EA as compared to controls shows, as an internal control, that the subjects correctly answered the questionnaires.

This study was conducted exclusively in patients with EA. One can argue that surgical damages, mainly towards vagal afferents, during EA repair may have influenced visceral pain processing. However, vagal afferents are believed to mediate physiological rather than harmful sensations transmitting information 
on nature and composition of the intestinal content and motility and contractile tension of the smooth muscle. ${ }^{14}$ It can also be argued that since children with EA may have "upper" gastrointestinal discomfort such as gastroesophageal reflux disease and dyspepsia, a risk of confusion between symptoms accompanying EA and those secondary to the neonatal stress induced by the EA surgery is possible. However, in this study, a majority of patients with EA presenting with a FGID diagnosis had mainly IBS or FAP (based on "lower digestive tract" complaints) enhancing the reliability of the results. Another pitfall is related to the number of subjects included, which may preclude the demonstration of differences between 2 groups of subjects by a possible type II error. We postulated that, to be clinically significant, the effect of neonatal stress on the incidence of FGID should be as strong as that of enteric infections in the development of IBS symptoms. ${ }^{10}$ Although this study was based on this hypothesis, we cannot rule out that a less profound effect of neonatal stress is present.

The putative role of early life events in the development of FGID comes from studies conducted in rodents indicating that the neonatal period is characterized by a very high susceptibility to stress leading to visceral hypersensitivity in adulthood. Neonatal colonic ${ }^{2}$ or gastric ${ }^{15}$ irritation, maternal deprivation ${ }^{3}$ and gastric suctioning ${ }^{16}$ have been shown to induce visceral (colonic $^{2,3,16}$ or gastric ${ }^{15}$ ) hypersensitivity in animal models. The mechanisms include peripheral ${ }^{3,4}$ and central sensitization ${ }^{2}$ and involve corticotrophin releasing factor, ${ }^{16}$ nerve growth factor ${ }^{17}$ and transient receptor potential vanilloid $1 .{ }^{18}$ Indeed, this period is a particularly sensitive window for experience-induced neuronal plasticity due to the ongoing maturation of sensory ${ }^{19}$ and pain processing. ${ }^{20}$

How do we explain the discrepancies between animal studies and the results of the present study? It is well known that early traumatic and painful experiences such as burns can induce long-term alterations in sensory and pain processing in children. ${ }^{21}$ Similarly, though different from neonatal stress, childhood trauma and abuse that are strongly associated to IBS in adults result in modifications in the hypothalamic-pituitary- adrenal axis. ${ }^{22}$ However, somatic pain researchers have also shown that long-term consequences of NICU admission are not only characterized by enhanced perceptual sensitization to prolonged painful stimulation but also by hypoalgesia to brief heat pain stimuli $^{23}$ suggesting that modifications of pain processing may occur in various directions. Ren et $\mathrm{al}^{24}$ corroborated this idea by showing in a rat model of neonatal local inflammation that global elevation of pain thresholds (ie, hypoalgesia) and excessive hyper- algesia upon re-inflammation in response to thermal and mechanical stimuli could occur both in the same animal. ${ }^{24}$ Furthermore, translational studies in FGID have shown that results obtained in animal models are only partially correlated to human symptoms. ${ }^{25}$ This is also illustrated in a study of somatic pain sensitivity to mechanical stimulation in children that was not significantly altered by painful neonatal experiences ${ }^{23}$ contrasting with results found in animals. ${ }^{24}$ All of these elements must be carefully taken into account when the replication of animal studies in the field of pain and visceral sensitivity is attempted on humans. Since visceral sensitivity processing is highly complex with involvement of peripheral and central nervous systems influenced by cognitive and psychological processes, it is easy to imagine that animal models only partially reflect human pathophysiology. In keeping with this idea, it has been shown that response to pain in school-aged children with previous experience in NICU was highly influenced by the mother's behavior. ${ }^{26,27}$

We conclude that severe neonatal stress (surgery, repeated physical aggressions and maternal separation) secondary to EA repair is not a clinically significant risk factor for pain-associated FGID later in life either in childhood or in adulthood. Invaluable pathophysiological knowledge gathered by animal models must be carefully interpreted in that context. To further understand the results of this study and the consequences of neonatal stress on visceral sensitivity, objective measurements of somatic pain, visceral sensitivity and psychological and cognitive assessment of the patients (and their parents) should be conducted. Measure of the response to stress is also warranted as suggested by recent work on epigenetic consequences of early life adverse events. ${ }^{28}$

\section{Acknowledgements}

We thank Lubomir Alexandrov, from the Applied Clinical Research Unit (URCA, CHU Sainte-Justine, Université de Montréal, Montréal, Québec, Canada) for his valuable help in statistical analysis. We also thank Dr Hubert Labelle and nurses from the Orthopedic Clinic at Ste-Justine Hospital for the recruitment of pediatric controls. Special thanks to Dr. Kelly Grzywacz for her help with editing the manuscript. Ugur Halac was involved in the conception and design of the study. He recruited pediatric patients in the Canadian team, collected clinical data, participated in the statistical analysis and he wrote the manuscript. Marine Revillion recruited pediatric patients in the French team and collected clinical data. She critically revised the manuscript. Laurent Michaud and Frédéric Gottrand recruited 
pediatric patients in the French team, and critically revised the manuscript. Christophe Faure designed the study. He recruited pediatric patients in the Canadian team, participated in the statistical analysis and wrote the manuscript.

\section{References}

1. Rasquin A, Di Lorenzo C, Forbes D, et al. Childhood functional gastrointestinal disorders: child/adolescent. Gastroenterology 2006; 130:1527-1537.

2. Al-Chaer ED, Kawasaki M, Pasricha PJ. A new model of chronic visceral hypersensitivity in adult rats induced by colon irritation during postnatal development. Gastroenterology 2000;119:1276-1285.

3. Barreau F, Ferrier L, Fioramonti J, Bueno L. Neonatal maternal deprivation triggers long term alterations in colonic epithelial barrier and mucosal immunity in rats. Gut 2004;53:501-506.

4. Lin $\mathrm{C}, \mathrm{Al}$-Chaer $\mathrm{ED}$. Long-term sensitization of primary afferents in adult rats exposed to neonatal colon pain. Brain Res 2003;971:73-82.

5. Anand KJS, Runeson B, Jacobson B. Gastric suction at birth associated with long-term risk for functional intestinal disorders in later life. J Pediatr 2004;144:449-454.

6. Bengtson MB, Rønning T, Vatn MH, Harris JR. Irritable bowel syndrome in twins: genes and environment. Gut 2006;55:1754-1759.

7. Saps M, Bonilla S. Early life events: infants with pyloric stenosis have a higher risk of developing chronic abdominal pain in childhood. J Pediatr 2011;159:551-554. e1.

8. Shaw-Smith C. Oesophageal atresia, tracheo-oesophageal fistula, and the VACTERL association: review of genetics and epidemiology. J Med Genet 2006;43:545-554.

9. Anonymous. Rome III diagnostic questionnaire for the pediatric functional GI disorders. In: Drossman DA, Corazziari E, Delvaux M, Spiller R, Talley NJ, Thompson WG, Whitehead WE, eds. The functional gastrointestinal disorders: Rome III. 3rd ed. McLean, VA: Degnon Associates, Inc. 2006:961-990.

10. Saps M, Pensabene L, Di Martino L, et al. Post-infectious functional gastrointestinal disorders in children. J Pediatr 2008;152:812816. e1.

11. Hyams JS, Burke G, Davis PM, Rzepski B, Andrulonis PA. Abdominal pain and irritable bowel syndrome in adolescents: a community-based study. J Pediatr 1996;129:220-226.

12. Castilloux J, Noble AJ, Faure C. Risk factors for short- and longterm morbidity in children with esophageal atresia. J Pediatr 2010; 156:755-760.

13. Chitkara DK, Rawat DJ, Talley NJ. The Epidemiology of childhood recurrent abdominal pain in Western countries: a systematic review. Am J Gastroenterol 2005;100:1868-1875.
14. Aziz Q, Thompson DG. Brain-gut axis in health and disease. Gastroenterology 1998;114:559-578.

15. Liu LS, Winston JH, Shenoy MM, Song GQ, Chen JD, Pasricha PJ. A rat model of chronic gastric sensorimotor dysfunction resulting from transient neonatal gastric irritation. Gastroenterology 2008;134: 2070-2079.

16. Smith C, Nordstrom E, Sengupta JN, Miranda A. Neonatal gastric suctioning results in chronic visceral and somatic hyperalgesia: role of corticotropin releasing factor. Neurogastroenterol Motil 2007;19: 692-699.

17. Barreau F, Cartier C, Ferrier L, Fioramonti J, Bueno L. Nerve growth factor mediates alterations of colonic sensitivity and mucosal barrier induced by neonatal stress in rats. Gastroenterology 2004;127: 524-534.

18. van den Wijngaard RM, Klooker TK, Welting O, et al. Essential role for TRPV1 in stress-induced (mast cell-dependent) colonic hypersensitivity in maternally separated rats. Neurogastroenterol Motil 2009;21:1107-e94.

19. Berardi N, Pizzorusso T, Maffei L. Critical periods during sensory development. Curr Opin Neurobiol 2000;10:138-145.

20. Fitzgerald M, Jennings E. The postnatal development of spinal sensory processing. Proc Natl Acad Sci USA 1999;96:7719-7722.

21. Wollgarten-Hadamek I, Hohmeister J, Demirakca S, Zohsel K, Flor $\mathrm{H}, \mathrm{Hermann} \mathrm{C}$. Do burn injuries during infancy affect pain and sensory sensitivity in later childhood? Pain 2009;141:165-172.

22. Videlock EJ, Adeyemo M, Licudine A, et al. Childhood trauma is associated with hypothalamic-pituitary-adrenal axis responsiveness in irritable bowel syndrome. Gastroenterology 2009;137:1954-1962.

23. Hermann C, Hohmeister J, Demirakca S, Zohsel K, Flor H. Long-term alteration of pain sensitivity in school-aged children with early pain experiences. Pain 2006;125:278-285.

24. Ren K, Anseloni V, Zou SP, et al. Characterization of basal and re-inflammation-associated long-term alteration in pain responsivity following short-lasting neonatal local inflammatory insult. Pain 2004; 110:588-596.

25. Mayer EA, Bradesi S, Chang L, Spiegel BM, Bueller JA, Naliboff BD. Functional GI disorders: from animal models to drug development. Gut 2008;57:384-404.

26. Mallen CD, Peat G, Thomas E, Croft PR. Is chronic pain in adulthood related to childhood factors? A population-based case-control study of young adults. J Rheumatol 2006;33:2286-2290.

27. Hohmeister J, Demirakça S, Zohsel K, Flor H, Hermann C. Responses to pain in school-aged children with experience in a neonatal intensive care unit: cognitive aspects and maternal influences. Eur J Pain 2009;13:94-101.

28. McGowan PO, Sasaki A, D'Alessio AC, et al. Epigenetic regulation of the glucocorticoid receptor in human brain associates with childhood abuse. Nat Neurosci 2009;12:342-348. 